\title{
PREVALÊNCIA DA DOR EM TRABALHADORES DA DIRETORIA DE SERVIÇOS DA FCT- UNESP
}

Amadeu Cavichiolli Prado; Giovana Rampazzo Teixeira ${ }^{1}$; Raul Fragoso Neto ${ }^{2}$; Robson Chacon Castoldi ${ }^{2}$; Olga Cristina de Mello Malheiro ${ }^{1}$.

${ }^{1}$ Universidade Estadual Paulista - FCT/UNESP, Departamento de Educação Física. ${ }^{2}$ Departamento de Fisioterapia, , Presidente Prudente - SP, Brasil. E-mail: giovana@ibb.unesp.br

\section{RESUMO}

A FCT-UNESP, Campus de Presidente Prudente, conta com 92 funcionários produtivos no labor e pertencentes à Diretoria de Serviços, onde o objetivo deste estudo foi analisar a quantidade e a intensidade da dor nos funcionários. Participaram da pesquisa 41 funcionários com média de idade de $47,51 \pm 7,90$ anos. $O$ instrumento para coleta de dados foi um questionário de topografia e intensidade da dor. O teste descritivo foi empregado na análise estatística dos dados. Os resultados indicaram em $71 \%$ da amostra 78 pontos de dores em 11 regiões corporais, com valores médios iguais ou acima do proposto pela escala de intensidade da dor. Conclui-se que os índices de dores elevados apontam para os Distúrbios Osteomusculares Relacionados ao Trabalho (DORT). No entanto, a proposta de um programa de Ginástica Laboral (GL) é um recurso na prevenção e pode promover melhor condição de trabalho e qualidade de vida para esta população.

Palavras-chave: dor, trabalhadores, transtornos traumáticos cumulativos, ginástica, prevenção primária.

\section{PREVALENCE OF PAIN IN WORKERS OF THE DIRECTORATE OF SERVICES FROM FCT- UNESP}

\begin{abstract}
The FCT - UNESP, Presidente Prudente, has 92 employees on the Direction of Services, so that the aim of this study was analyze the amount and intensity of pain in these employees. Fourty one employees participated with mean age of $47.51 \pm 7,90$ years. The instrument of data collection was a questionnaire of pain topography and intensity. It was used a descriptive test for statistical analysis. The results indicated that $71 \%$ of the employees have 78 spots of pain in 11 regions, with average values equal to or above the scale proposed for intensity of pain. It is conclude that the high index of pain suggest Cumulative Trauma Disorders. However, the proposal of a program of workplace physical activity program is an important resource in the prevention and can promote better working condition and quality of life for this population.
\end{abstract}

Keywords: pain, workers, cumulative trauma disorders, gymnastics, primary prevention.

\section{INTRODUÇÃO}

Atualmente, o trabalho exige a maior parte de tempo que indivíduo possui. Por esse motivo, pessoas têm cada vez menos tempo para o lazer e atividades físicas que Ihe tragam benefícios. Esse comportamento contribui para aumento do sedentarismo e o aparecimento das 
doenças crônicas não transmissíveis como: hipertensão arterial, diabetes, estresse e doenças osteomusculares ${ }^{1}$.

Atualmente, a alta incidência dos distúrbios osteomusculares está associada com a tecnologia e a informatização, forçando os trabalhadores a permanecerem em um trabalho estático e repetitivo durante várias horas ao dia; gerando assim, a fadiga muscular e grande desconforto físico e mental ${ }^{2}$.

Os DORT são lesões que acometem tanto os músculos e tendões como nervos dos membros inferiores e, com maior frequência, nos membros superiores. Além disso, afeta regiões como pescoço e tronco. Desta forma, evoluem para inflamações crônicas com consequências funcionais ${ }^{3}$.

Para Couto et al., ${ }^{4}$ os DORT são transtornos funcionais e mecânicos com lesões de músculos, tendões, fáscias, nervos, bolsas articulares e pontas ósseas nos membros superiores ocasionados pela utilização biomecânica incorreta, o que resultam em dor, fadiga, queda da performance no trabalho e incapacidade temporária.

Os distúrbios são, portanto, decorrentes dos fatores físicos, psicológicos, sociais, biomecânicos e de posto e/ou organização de trabalho. Os principais fatores biomecânicos correspondem à repetitividade, força excessiva, postura errada, compressão e vibração mecânica ${ }^{5}$.

A Organização Internacional do Trabalho (OIT) relata que os países arcam com custos médios equivalentes a $4 \%$ de seus PIBs a cada ano em decorrência de acidentes, tratamentos de doenças, lesões e incapacidades relacionadas ao trabalho ${ }^{5}$. Segundo Barboza et al., ${ }^{6}$ estima-se que $60 \%$ dos afastamentos de trabalhadores são decorrência de DORT, o que se torna uma preocupação por parte dos funcionários e um desafio para as empresas.

A preocupação, devido à grande incidência das doenças ocupacionais, gerou métodos para que fosse possível quantificar e analisar a saúde do trabalhador e incentivar programas de promoção de saúde, que proporcionem redução desses afastamentos provocados por tais distúrbios. Assim, o objetivo desta pesquisa foi de analisar e quantificar a porcentagem de trabalhadores da Diretoria de Serviços da FCT-UNESP, Campus de Presidente Prudente, que sentem dor, buscando identificar a intensidade de dor e mapear as regiões do corpo frequentemente acometidas.

\section{MATERIAIS E MÉTODOS}

Caracterização da Pesquisa 
Esta pesquisa caracteriza-se como um estudo descritivo, o qual tem como objetivo descrever a quantidade e a intensidade das dores em funcionários da FCT-UNESP e está de acordo com o Comitê de Ética (processo no43/2010).

\section{População e Amostra}

A população desta pesquisa compõe-se de funcionários produtivos no labor, pertencentes à Diretoria de Serviços da FCT-UNESP, Campus de Presidente Prudente. A coleta dos dados foi realizada no período de 2010 a 2012. A amostra constituiu-se de 41 funcionários do sexo masculino com a média de idade de 47,51 \pm 7,90 anos.

\section{Instrumento de Coletas de Dados}

O instrumento utilizado para avaliar a dor dos funcionários é o questionário de topografia e intensidade da dor, auto-administrado, com indicação de figuras da região anterior e posterior do corpo $^{7}$.

\section{Analise Estatística}

Para a determinação dos valores mínimos, máximos, médias e o desvio padrão, utilizou-se a análise descritiva contida no Software SPSS Statistics 17.0. Para tal, foram classificadas as regiões corporais acometidas pela dor com suas intensidades.

\section{RESULTADOS}

Dos 41 funcionários que responderam o questionário, 29\% relataram não sentir nenhuma dor, mas 71\% apontaram algum ponto de dor. Foram encontrados 78 pontos de dores nas 11 regiões corporais dos servidores que apresentaram algum tipo de dor (Tabela 1).

As regiões corporais onde os maiores valores prevaleceram com maior intensidade foram os punhos, as mãos e o quadril. Valores mínimos de 5 e máximos de 8 eram para punhos e mãos e 10 para quadril, sendo estes, valores elevados. Já a região corporal com menor valor foi o pescoço, referente a intensidade da dor.

Tabela 1. Valores mínimos, máximos e médios \pm desvio padrão (DP) da intensidade de dor corporal para cada região corporal 


\begin{tabular}{cccc}
\hline & Mínimo & Máximo & Média \pm DP \\
\hline Pescoço & 3 & 7 & $5 \pm 1,67$ \\
Ombros & 4 & 10 & $6,8 \pm 2,26$ \\
Cotovelos & 5 & 8 & $7,2 \pm 1,30$ \\
Braços & 4 & 8 & $7,1 \pm 1,60$ \\
Punhos e Mãos & 5 & 8 & $7,8 \pm 1,30$ \\
Coluna Dorsal & 3 & 10 & $7,2 \pm 2,62$ \\
Coluna Lombar & 5 & 10 & $5,4 \pm 1,65$ \\
Quadril & 5 & 10 & $7,8 \pm 1,78$ \\
Pernas & 3 & 8 & $6 \pm 2,00$ \\
Joelhos & 2 & 10 & $6,7 \pm 3,03$ \\
\hline
\end{tabular}

As regiões corporais mais acometidas são ombros com $51,7 \%$ e coluna lombar com $48,2 \%$ dos funcionários, seguidos da coluna torácica e joelhos, ambos com $24,1 \%$. Já as regiões menos acometidas são os tornozelos e os pés, com apenas 6,8\%. É importante ressaltar que as regiões corporais como a coluna lombar, onde o número de funcionários acometidos pela dor é o segundo maior $(48,2 \%)$, tem seus valores médios de intensidade da dor como a segunda menor $(5,4 \pm 1,65)$. Figura 2. Porcentagem de funcionários para cada região corporal

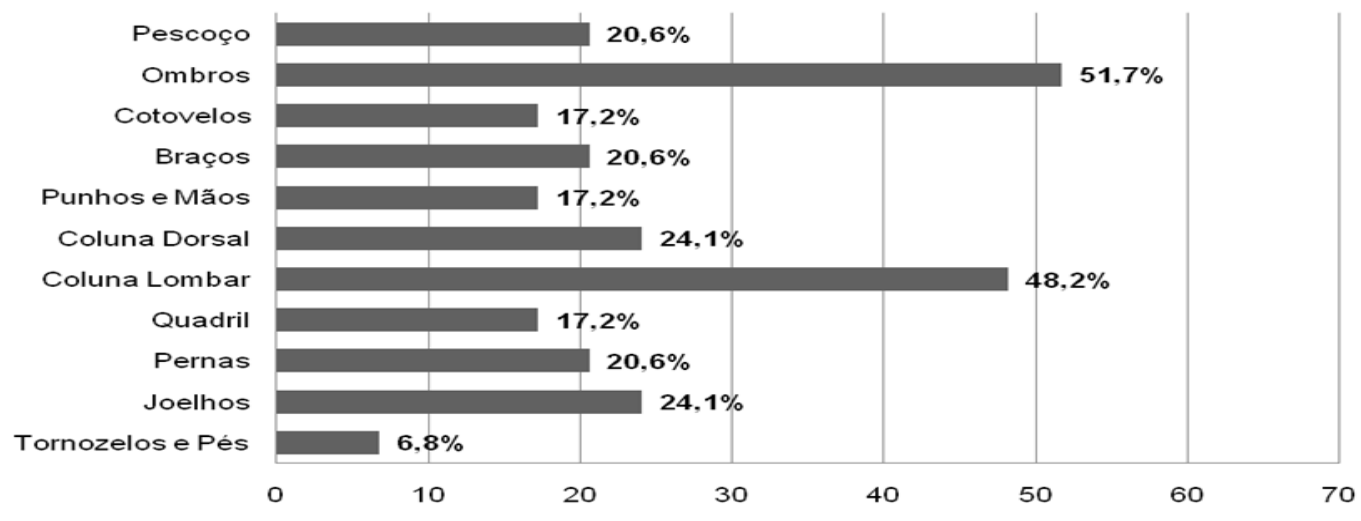

\section{DISCUSSÃO}

Os DORT são doenças ocupacionais de origem multicausal e diversos fatores influenciam para o seu aparecimento. Dentre eles, podem-se destacar os ambientais (frio, calor, iluminação), individuais (genética) psicológicos (estresse, insatisfação), ergonômicos (móveis inadequados, postura) e, principalmente organizacionais (execução das tarefas) ${ }^{8}$.

Nesta pesquisa, foram identificados 78 pontos de dor nas 11 regiões corporais dos 29 funcionários (71\%) avaliados com dor, o que significa uma média de 2,68 pontos de dor por funcionário. Esses dados apontam uma grande incidência de dores e risco de DORT em funcionários da FCT-UNESP.

Constatamos que os ombros foram as regiões mais afetadas pela dor. Movimentos repetitivos são os causadores de grande número de doenças que afetam os membros superiores ${ }^{9}$. 
Uma segunda área mais afetada com os movimentos repetitivos foi a coluna lombar. Devido ao esforço realizado diariamente, os músculos da região lombar trabalham permanentemente sempre que algum movimento de tronco é realizado, sofrendo movimentos bruscos, quedas e sobrecargas musculares ${ }^{10}$.

A dor é um processo complexo que tem como objetivo alertar o organismo a respeito da agressão. Pode-se classificar a dor em duas categorias: a dor aguda e a dor crônica. A aguda é a que geralmente tem um ponto identificável e duração limitada. Já a dor crônica ocorre quando os mecanismos de inibição da dor não funcionam adequadamente, assim se tornando contínua e consequentemente uma doença ${ }^{11}$.

Desta forma, um dos métodos para a prevenção dos DORT é o das atividades físicas executadas no ambiente de trabalho e conhecidas como ginástica laboral (GL). Kolling et al., ${ }^{12}$ por meio de um estudo comparativo, demonstrou que a pausa ativa reduz significativamente o índice de fadiga periférica. A fadiga tem sido sugerida como um mecanismo de defesa contra possíveis efeitos deletérios em determinadas funções orgânicas e celulares, protegendo assim a integridade da fibra muscular esquelética, órgãos e tecidos, como um todo ${ }^{13}$.

A GL é um conjunto de atividades físicas com a finalidade de preparar o organismo para o trabalho físico, promover a manutenção e normalização do tônus muscular e também compensar e relaxar qualquer esforço repetitivo nos músculos, articulações, tendões e ligamentos, ocorridos no período de trabalho ${ }^{14}$.

Os funcionários da FCT-UNESP apresentaram preocupantes valores de dor, pois, em $71 \%$ da amostra, foram presenciadas mais de duas regiões corporais afetadas e, em todas elas, tiveram valores médios iguais ou acima do moderado (5) na escala de intensidade da dor. Isso mostra que os riscos dos DORT estão presentes nesta população e que a prevenção é uma opção existente para seu manejo. A implantação de um programa GL poderá servir como recurso para o problema da empresa e dos funcionários da FCT-UNESP, pois a GL é um método eficaz na prevenção das doenças ocupacionais como as DORT, na diminuição das dores corporais, além da melhoria das condições de trabalho e qualidade de vida para esta população.

\section{CONCLUSÃO}

Conclui-se que $71 \%$ dos entrevistados relataram algum tipo de dor, sendo as regiões do ombro e coluna lombar acometidas com maior frequência. 


\section{REFERÊNCIAS}

1. Rego RA. et al. Fatores de risco para doenças crônicas não-transmissíveis: inquérito domiciliar no município de São Paulo. Revista de Saúde Pública, mar 1990; v. 24, n. 4, p. 277 - 285. http://dx.doi.org/10.1590/S0034-89101990000400005

2. Pereira ER. Fundamentos de ergonomia e fisioterapia do trabalho. 2. ed. Rio de Janeiro: Ed. Taba Cultural, 2001.

3. Moraes MA. Miguez SA. LER/DORT: Prevenção, tratamento e noções básicas de ergonomia. Campinas: Fernandes Fisioterapia, 1998 (Apostila de atualização do curso).

4. Couto HA.; Nicoletti SJ, Lech O. Como gerenciar a questão das LER/DORT. Belo Horizonte: Ed. Ergo, 1998.

5. Andrade AL. LER: uma visão da doença. Revista Fenacon, v.5, n. 54, p. 14-17, jun. 2000.

6. Barboza MCN, Milbrath VM, Bielemann VM, Siqueira HCH. Doenças osteomusculares relacionadas ao trabalho (DORT) e sua associação com a enfermagem ocupacional. Rev Gaúcha Enfermagem, Porto Alegre (RS) 2008 dez;29(4):633-8.

7. Mendes RA, Leite N. Ginástica laboral: princípios e aplicações práticas. 2. ed. Barueri: Manole, 2008.

8. MINISTÉRIO DA SAÚDE DO BRASIL, Organização Pan-Americana da Saúde/Brasil. Doenças relacionadas ao trabalho. Série A, normas e manuais técnicos, 2011; n.114.

9. Yassi A. Repetitive strain injuries. Occupational Medicine. 1997; v. 349, p. 943 - 947.

10. Bertolini E. A ginástica laboral como caminho para a qualidade de vida no trabalho.

Trabalho de conclusão de curso (Graduação em Educação Física) - Universidade do Contestado, Concórdia, 1999.

11. Lima V. Ginástica laboral atividade física no ambiente de trabalho. 3. ed. São Paulo: Phorte, 2007.

12. Kolling A. Ginástica laboral compensatória. Revista Brasileira de Educação Física e Desportos, Brasília, 1982; v.12, n.44, p. 20-23, 1982.

13. Enoka RM, Duchateau J. Muscle fatigue: what, why and how it influences muscle function. Journal of Physiology, 2008; v. 586, n. 1, p. 11-23. DOI: 10.1113/jphysiol.2007.139477

14. Moraes C, Delbin MA. Por que implantar um programa de ginástica laboral na empresa? Revista de Administração, Espírito Santo do Pinhal, 2005; v.5, n.9, p. 7-9. 